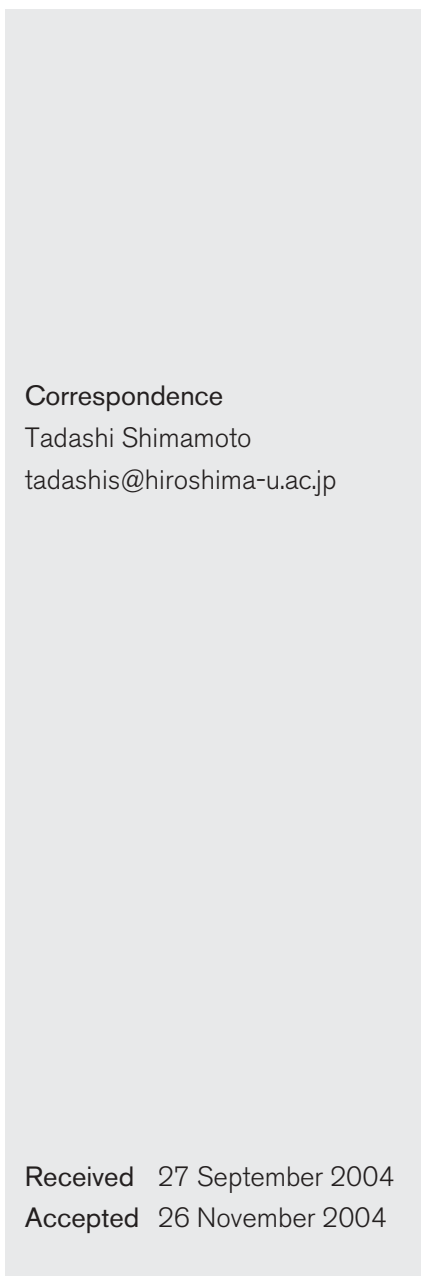

\section{Molecular characterization of a multidrug-resistant strain of enteroinvasive Escherichia coli O164 isolated in Japan}

\author{
Ashraf M. Ahmed, ${ }^{1}$ Shin-ichi Miyoshi, ${ }^{2}$ Sumio Shinoda ${ }^{2}$ \\ and Tadashi Shimamoto ${ }^{1}$ \\ ${ }^{1}$ Laboratory of Food Microbiology and Hygiene, Graduate School of Biosphere Science, Hiroshima \\ University, Higashi-Hiroshima 739-8528, Japan \\ ${ }^{2}$ Graduate School of Natural Science and Technology, Okayama University, Okayama 700-8530, \\ Japan
}

\begin{abstract}
Enteroinvasive Escherichia coli (EIEC) O164 strain RIMD05091045 was isolated from a travelling patient suffering from diarrhoea at the Osaka airport quarantine facility in Japan. The strain showed multidrug resistance against streptomycin, spectinomycin, co-trimoxazole (trimethoprim/ sulfamethoxazole) and ampicillin, and reduced susceptibility to ciprofloxacin. Molecular characterization of the multidrug-resistance phenotype revealed the presence of a class 1 integron containing three genes, a dihydrofolate reductase type XII gene, dfrXII, which confers resistance to trimethoprim, an aminoglycoside adenyltransferase gene, aadA2, which confers resistance to streptomycin and spectinomycin, and an ORF of unknown function. Southern blot hybridization and conjugation experiments showed that the class 1 integron was located on a transferable plasmid that was less than $90 \mathrm{~kb}$ in size. The resistance of EIEC O164 to ampicillin was found to be due to the presence of TEM-1 $\beta$-lactamase. On the other hand, a single mutation that has not previously been described, P158-to-S, was detected downstream of the quinolone-resistance-determining region of parC of topoisomerase IV and may be responsible for the reduced susceptibility to ciprofloxacin in this strain.
\end{abstract}

\section{INTRODUCTION}

Bacillary dysentery, which is mainly caused by Shigella and enteroinvasive Escherichia coli (EIEC), is responsible for a substantial proportion of acute diarrhoeal diseases worldwide (Taylor et al., 1986). EIEC outbreaks are usually foodor water-borne (Marier et al., 1973; Snyder et al., 1984). However, outbreaks of EIEC diarrhoea through person-toperson transmission have also been reported (Harris et al., 1985). E. coli serotype O164 is the most prevalent serotype among the EIEC in Bulgaria and is annually isolated in sporadic, as well epidemic, cases (Todorova et al., 1990). In Japan, EIEC serotype O164 is rare and was responsible for a case of food poisoning in 1992 (Yamamura et al., 1992). Epithelial cell invasion is a key virulence factor for EIEC, and

Abbreviations: CCCP, carbonyl cyanide m-chlorophenylhydrazone; EIEC, enteroinvasive Escherichia coli; ETEC, enterotoxigenic Escherichia coli; MDR, multidrug resistance; ORDR, quinolone resistance-determining region.

The GenBank/EMBL/DDBJ accession numbers for the class 1 integron sequence, including the $d f r X I l$ and aadA2 genes and an unknown ORF, and the blaTEM-1 gene sequence of EIEC 0164 strain RIMD05091045 are AB154407 and AB194682, respectively. thus dysentery is characterized by painful abdominal cramps and frequent defecation of blood and mucus attributed to the penetration and destruction of colonic epithelia (Nataro \& Kaper, 1998). EIEC strains are closely biochemically, genetically and pathogenetically related to Shigella species (Nataro \& Kaper, 1998).

Antimicrobial resistance associated with diarrhoea is an issue of great significance for public health at the global level. Moreover, it is of particular concern if the causative agents have multidrug resistance (MDR). In the last two decades, MDR phenotypes have spread widely among Gram-negative bacteria. One of the most important tools for this spread is the recently discovered genetic element known as an integron (Jones et al., 1997).

Integrons are natural genetic engineering systems that incorporate circularized ORFs, called gene cassettes, and convert them into functional genes (Rowe-Magnus \& Mazel, 2001). The most notable gene cassettes identified within integrons are those conferring resistance to antibiotics (Rowe-Magnus \& Mazel, 2001). Integron platforms are incapable of self-transposition, but this defect is often complemented through association with an insertion 
sequence, transposons and/or conjugative plasmids that can serve as vehicles for intra- and inter-species transmission of genetic material (Rowe-Magnus et al., 2002).

Resistance to ampicillin in E. coli is primarily mediated by $\beta$ lactamases, which hydrolyse the $\beta$-lactam ring and thus inactivate the antibiotic (Livermore, 1995). Many different $\beta$-lactamases have been described, but TEM-, SHV- and OXA-type $\beta$-lactamases are the most predominant (Bradford, 2001).

Fluoroquinolones are broad-spectrum antibiotics used for the treatment of a wide range of infections. Ciprofloxacin is one of the recently discovered fluoroquinolones that are recommended by the WHO for treatment of bacillary dysentery (WHO, 1995). One of the main mechanisms of fluoroquinolone resistance in bacteria is point mutations, which primarily occur in highly conserved regions [quinolone-resistance-determining regions (QRDRs)] of DNA gyrase (encoded by gyrA and $g y r B$ ) and topoisomerase IV (encoded by parC and parE) (Piddock, 1999). These mutations result in alteration of the target proteins, namely DNA gyrase and topoisomerase IV. However, many novel mutations located outside QRDRs have recently been discovered in gyrA (Chaudhry et al., 2002; Fournier \& Hooper, 1998; Ince \& Hooper, 2000) and parC (Bebear et al., 2003; Chaudhry et al., 2002; Fendukly et al., 2003; Trees et al., 1998). The mutations related to parC are responsible for resistance or reduced susceptibility to ciprofloxacin. On the other hand, efflux pumps are also considered to be important mechanisms for fluoroquinolone resistance in bacteria (Webber \& Piddock, 2003). For example, the AcrB efflux pump plays a major role in the antibiotic-resistance pheno- type of E. coli and addition of carbonyl cyanide $m$-chlorophenylhydrazone (CCCP) abolishes this effect (Webber \& Piddock, 2001).

Although there is considerable information concerning the epidemiology and virulence of EIEC, little is known about the molecular basis of antimicrobial resistance of this type. The aim of this study was to characterize the molecular bases of resistance of the multidrug-resistant EIEC O164 strain RIMD05091045.

\section{METHODS}

Bacterial strains. EIEC O164 strain RIMD05091045 was isolated from a travelling patient suffering from diarrhoea at the Osaka airport quarantine facility in Japan and sent to the Osaka Prefectural Public Health Institute, Japan. EIEC O164 strain RIMD05091045 was then stored at the Research Institute for Microbial Diseases, Osaka University, Japan, until use. The E. coli isolate was identified by standard procedures (Bopp et al., 1999) and serotyping was performed according to the method of Orskov \& Orskov (1984).

Detection of the invasion plasmid antigen $\mathbf{H}$ gene of EIEC. The invasion plasmid antigen $\mathrm{H}$ gene $(\mathrm{ipaH})$ is the main virulence determinant of EIEC. PCR was used to detect this gene in EIEC O164 strain RIMD05091045 using the primers ipaIII and ipaIV (Table 1) as previously described (Toma et al., 2003).

Antimicrobial susceptibility testing. The MICs of the antibiotics were determined by the NCCLS broth microdilution method. The MICs for EIEC O164 strain RIMD05091045 were estimated for the following antibiotics supplied by different commercial companies: ampicillin, chloramphenicol, ciprofloxacin, gentamicin, kanamycin, streptomycin, spectinomycin, tetracycline, co-trimoxazole (trimethoprim/sulfa-

Table 1. Oligonucleotides used for PCR and DNA sequencing

\begin{tabular}{|c|c|c|c|}
\hline Primer & Nucleotide sequence ( $5^{\prime}$ to $\left.3^{\prime}\right)$ & Target size & Reference or accession no. \\
\hline ipaIII & GTTCCTTGACCGCCTTTCCGATACCGTC & $619 \mathrm{bp}$ & Toma et al. (2003) \\
\hline ipaIV & GCCGGTCAGCCACCCTCTGAGAGTAC & & \\
\hline $5^{\prime}-\mathrm{CS}$ & GGCATCCAAGCAGCAAG & - & Lévesque et al. (1995) \\
\hline $3^{\prime}-\mathrm{CS}$ & AAGCAGACTTGACCTGA & & \\
\hline EIEC-intF2 & CGCCAAAATGCTGCGCATT & - & This study \\
\hline EIEC-intR2 & AGATCATTGAGCAATGCTCG & & \\
\hline TEM-F & ATAAAATTCTTGAAGACGAAA & $1080 \mathrm{bp}$ & Weill et al. (2004) \\
\hline TEM-R & GACAGTTACCAATGCTTAATC & & \\
\hline SHV-F & TTATCTCCCTGTTAGCCACC & $795 \mathrm{bp}$ & Weill et al. (2004) \\
\hline SHV-R & GATTTGCTGATTTCGCTCGG & & \\
\hline OXA-F & TCAACTTTCAAGATCGCA & $591 b p$ & J02967 \\
\hline OXA-R & GTGTGTTTAGAATGGTGA & & \\
\hline GYRA-F & ATGAGCGACCTTGCGAGAGAAATTACACCG & $630 \mathrm{bp}$ & AE005455 \\
\hline GYRA-R & TTCCATCAGCCCTTCAATGCTGATGTCTTC & & \\
\hline GYRB-F & GGACAAAGAAGGCTACAGCA & $882 \mathrm{bp}$ & D87842 \\
\hline GYRB-R & TGCGTCGCGTTGTACTCAGA & & \\
\hline PARC-F & ATGAGCGATATGGCAGAGCG & $589 \mathrm{bp}$ & M58408 \\
\hline PARC-R & CGATTAATGCGATTGCCGCCTGA & & \\
\hline PARE-F & GGAAGATATCTGGGATCGCTG & $590 \mathrm{bp}$ & AE005533 \\
\hline PARE-R & AACAAAGCGCAGAGCAGTGTAG & & \\
\hline
\end{tabular}


methoxazole), neomycin and cefotaxime. MIC breakpoints were evaluated according to NCCLS guidelines (NCCLS, 2002).

\begin{abstract}
Bacterial DNA preparation, and PCR and DNA sequencing of the class 1 integron. The preparation of the bacterial DNA template and PCR conditions for detection of the class 1 integron were as previously described (Zhao et al., 2001). The class 1 integron primers 5'-CS and 3'-CS (Table 1; Lévesque et al., 1995), which amplify the region between $5^{\prime}$-CS and $3^{\prime}$-CS, were used. The reaction products were subjected to electrophoresis in a $1.0 \%$ agarose gel, stained with ethidium bromide and visualized under UV light. The PCR fragment was then purified from the agarose gel using a QIAquick Gel Extraction Kit (QIAGEN). Both DNA strands of the PCR product were sequenced using an ABI automatic DNA sequencer (model 373, Perkin-Elmer). According to the preliminary DNA sequencing results, two other primers, EIEC-intF2 and EIEC-intR2 (Table 1), were designed. These primers were located within the PCR fragment and used for complete sequencing of both strands of the whole integron segment.
\end{abstract}

Plasmid isolation, probe preparation and Southern blot hybridization. Plasmids were isolated from EIEC O164 strain RIMD05091045 using the alkaline lysis method described by Sambrook \& Russell (2001). After agarose gel electrophoresis, the DNA fragments in the gel were transferred onto Hybond- $\mathrm{N}^{+}$nylon membranes (Amersham Biosciences) according to the manufacturer's instructions. The DNA fragment containing the whole integron $(1 \cdot 8 \mathrm{~kb})$ was amplified by PCR using the integron primers $5^{\prime}$-CS and $3^{\prime}$-CS (Table 1) and purified as described above. The purified fragment waslabelled with alkaline phosphatase using the AlkPhos Direct Labelling System (Amersham Biosciences), and subsequently used as a DNA probe. All hybridization steps were carried out according to the manufacturer's protocol. The hybridization was performed at $55^{\circ} \mathrm{C}$ for $12 \mathrm{~h}$, and the hybridized DNA was detected using the CDP-Star chemiluminescent signal generation system (Amersham Biosciences) according to the manufacturer's instructions.

Conjugation experiments. Direct transfers of plasmids carrying resistance genes were performed by mating the donor strain, EIEC O164 strain RIMD05091045, with a rifampicin-resistant mutant of E. coli HB101 obtained in vitro (Sambrook \& Russell, 2001) as the recipient strain at $37^{\circ} \mathrm{C}$ in solid and liquid Mueller-Hinton medium. Transconjugants were selected on Mueller-Hinton agar containing $200 \mu \mathrm{g}$ rifampicin $\mathrm{ml}^{-1}, 50 \mu \mathrm{g}$ streptomycin $\mathrm{ml}^{-1}$ and $25 \mu \mathrm{g}$ trimethoprim $\mathrm{ml}^{-1}$.

Screening for $\boldsymbol{\beta}$-lactamase-encoding genes. EIEC O164 strain RIMD05091045 was tested for TEM, SHV and OXA $\beta$-lactamaseencoding genes by PCR as previously described (Weill et al., 2004). Universal primers for the TEM, SHV and OXA families were used (Table 1).

PCR amplification and DNA sequencing of the QRDRs of DNA gyrase and topoisomerase IV. PCR and DNA sequencing techniques were used to detect and identify mutations in the DNA gyrase ( $g y r A$ and gyrB) and topoisomerase IV (parC and parE) genes of EIEC O164 strain RIMD05091045. The oligonucleotide primers used for PCR amplification and DNA sequencing are described in Table 1. PCR amplification of the QRDRs was initiated by denaturation at $95{ }^{\circ} \mathrm{C}$ for $5 \mathrm{~min}$, followed by 30 cycles of denaturation for $45 \mathrm{~s}$ at $95^{\circ} \mathrm{C}$, primer annealing for $20 \mathrm{~s}$ at $54{ }^{\circ} \mathrm{C}$ and extension for $1 \mathrm{~min}$ at $72{ }^{\circ} \mathrm{C}$, and then a final extension at $72{ }^{\circ} \mathrm{C}$ for $10 \mathrm{~min}$. The amplified products were visualized, purified and sequenced as described above for the class 1 integron.

Determination of the efflux pump mechanism. The efflux pump activity was roughly checked in EIEC O164 strain RIMD05091045 by determination of the MIC of ciprofloxacin in the presence and absence of efflux pump inhibitor $100 \mu \mathrm{M}$ CCCP (Sigma). This experiment was performed twice to ensure reproducibility.
Computer analysis of the sequence data. A similarity search was carried out using the BLAST program available at the NCBI BLAST homepage (http://www.ncbi.nlm.nih.gov/blast/).

\section{RESULTS AND DISCUSSION}

\section{Multidrug-resistance phenotype of EIEC 0164 strain RIMD05091045}

Diarrhoea caused by multidrug-resistant bacteria is an important public health problem among children and adults in developing countries, and represents a research priority of the Diarrhoeal Disease Control programme of the World Health Organization. In this study, we identified a multidrug-resistant bacterium, EIEC O164 strain RIMD05091045, which was isolated from a sporadic case of diarrhoea in Japan. This strain displayed a MDR phenotype against streptomycin (MIC, $64 \mu \mathrm{g} \mathrm{ml}^{-1}$ ), spectinomycin (MIC, $64 \mu \mathrm{g} \mathrm{ml}^{-1}$ ), ampicillin (MIC, $32 \mu \mathrm{g} \mathrm{ml}^{-1}$ ) and co-trimoxazole (trimethoprim/sulfamethoxazole) (MIC, 16/304 $\mu \mathrm{g}$ $\mathrm{ml}^{-1}$ ), and a reduced susceptibility to ciprofloxacin (MIC, $2 \mu \mathrm{g} \mathrm{m}^{-1}$ ). On the other hand, it was susceptible to tetracycline (MIC, $0.5 \mu \mathrm{g} \mathrm{ml}^{-1}$ ), neomycin (MIC, $1 \mu \mathrm{g} \mathrm{ml}^{-1}$ ), gentamicin (MIC, $0.5 \mu \mathrm{g} \mathrm{ml}^{-1}$ ), kanamycin (MIC, $2 \mu \mathrm{g}$ $\mathrm{ml}^{-1}$ ), chloramphenicol (MIC, $2 \mu \mathrm{g} \mathrm{ml}^{-1}$ ) and cefotaxime (MIC, $0 \cdot 125 \mu \mathrm{g} \mathrm{ml}^{-1}$ ). The MDR phenotype of EIEC O164 strain RIMD05091045 is of great clinical significance because trimethoprim/sulfamethoxazole, ampicillin and ciprofloxacin are among the antibiotics recommended by the WHO for treatment of bacillary dysentery (WHO, 1995). Therefore, the selection of recommended antimicrobials for treatment of bacillary dysentery should be based on recent susceptibility tests.

\section{Gene cassettes of the class 1 integron from EIEC 0164 strain RIMD05091045}

The spread of MDR among Gram-negative bacteria is mainly due to the presence of class 1 integrons (Jones et al., 1997). In order to assess the relationship between the resistance phenotype of EIEC O164 strain RIMD05091045 and the presence of the class 1 integron, the strain was tested using PCR. The PCR results revealed the presence of a class 1 integron with an amplicon size of 1847 bp (Fig. 1).

DNA sequencing of the class 1 integron identified three ORFs (Fig. 2). The first ORF is a dihydrofolate reductase type XII gene, $d f r X I I$, which confers resistance to trimethoprim, the second ORF has an unknown product and the third is an aminoglycoside adenyltransferase gene, aadA2, which confers resistance to streptomycin and spectinomycin.

Several studies have reported the presence of class 1 integrons among clinical isolates of different types of E. coli. For example, class 1 integrons were reported to be associated with MDR in enterotoxigenic E. coli (ETEC) (Maynard et al., 2003), enterohaemorrhagic E. coli (Zhao et al., 2001), and enteroaggregative E. coli and EIEC (Gassama et al., 2004). In Japan, we recently characterized a class 1 integron in MDR ETEC O159 (Ahmed \& Shimamoto, 2004). 


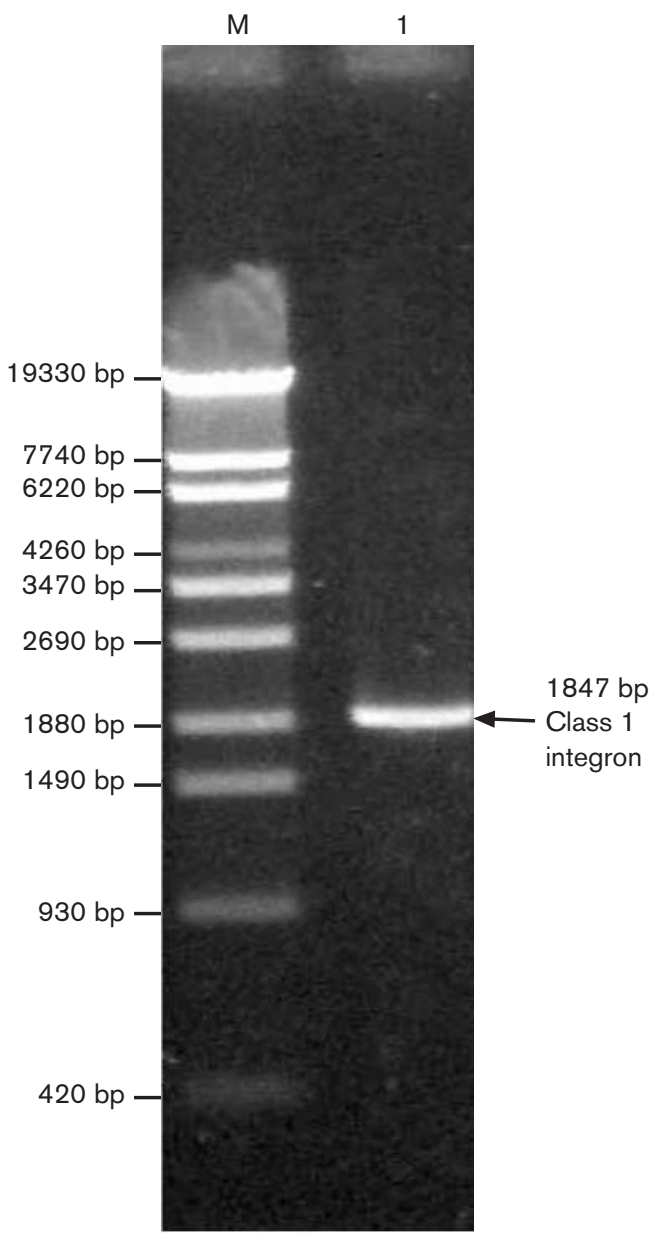

Fig. 1. Agarose gel electrophoresis of the PCR product (lane 1) of the class 1 integron from EIEC 0164 strain RIMD05091045. Lane M contains $\lambda$ DNA digested with Styl.

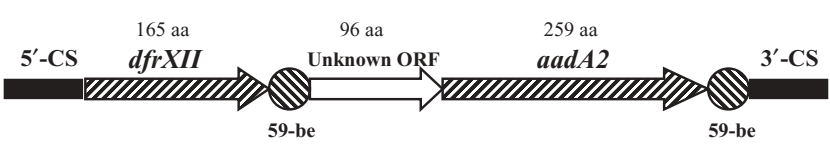

Fig. 2. Organization of the class 1 integron of EIEC 0164 strain RIMD05091045. Black boxes, 5' and $3^{\prime}$ conserved sequences; hatched arrows, antibiotic gene cassettes of the dihydrofolate reductase type 12 gene $(d f r X I)$, which confers resistance to trimethoprim, and aminoglycoside adenyltransferase type 2 gene ( $\operatorname{adA2}$ ), which confers resistance to streptomycin and spectinomycin; hatched circles, the 59-base elements (59-be) of the gene cassettes; white arrow, ORF of unknown function.

\section{The class 1 integron is located on a transferable plasmid}

Southern blot hybridization was used to determine the location of the class 1 integron in EIEC O164 strain RIMD05091045. Many plasmids of different sizes were isolated from EIEC O164 strain RIMD05091045 (Fig. 3), but a positive hybridization signal was only detected in a plasmid of just under $90 \mathrm{~kb}$ in size (Fig. 3). This plasmid was transferred from EIEC O164 strain RIMD05091045 to E. coli HB101 by conjugation and the E. coli transconjugant showed a resistance phenotype to streptomycin and trimethoprim on plates. The presence of class 1 integrons on transferable plasmids is considered to be the main mechanism for the rapid spread of MDR phenotypes among Gram-negative bacteria (Jones et al., 1997; Rowe-Magnus \& Mazel, 2001; Rowe-Magnus et al., 2002).

\section{Ampicillin-resistance gene determinant}

EIEC O164 strain RIMD05091045 was investigated for the presence of $\beta$-lactamase-encoding genes. PCR and DNA sequencing showed that the ampicillin resistance was due to the presence of a TEM-1 $\beta$-lactamase gene. TEM- 1 is the most commonly encountered $\beta$-lactamase in Gram-negative bacteria, and up to $90 \%$ of ampicillin resistance in E. coli is due to the production of TEM-1 (Livermore, 1995). To our knowledge, this is the first report of identification of a TEM $\beta$-lactamase gene in EIEC.

\section{A novel point mutation in parC of topoisomerase IV}

Fluoroquinolones mainly work through inhibition of the DNA-supercoiling activities of DNA gyrase and/or topoisomerase IV genes (Piddock, 1999). Resistance mutations occur in a short discrete segment termed the quinoloneresistance-determining region (QRDR) of DNA gyrase ( $g y r A$ and $g y r B$ genes) and the analogous sequences of topoisomerase IV (parC and parE genes). In addition, other resistance mutations have been detected outside the QRDRs (Bebear et al., 2003; Chaudhry et al., 2002; Fendukly et al., 2003; Fournier \& Hooper, 1998; Ince \& Hooper, 2000; Trees et al., 1998).

In this study, EIEC O164 strain RIMD05091045 displayed a reduced susceptibility to ciprofloxacin (MIC, $2 \mu \mathrm{g} \mathrm{ml}^{-1}$ ), which is considered to be one of the most suitable drugs for treatment of bacillary dysentery (WHO, 1995). In order to determine the basis of the reduced susceptibility to ciprofloxacin in this strain, we amplified and sequenced the QRDRs of gyrA, gyrB, parC and parE (data not shown). The sequencing results revealed only a single mutation in parC, $\mathrm{P} 158$-to-S. This mutation has not been reported before and is located downstream of the QRDR. No mutations were detected in $g y r A$, gyrB or parE. Associations between mutations within parC only and resistance or reduced susceptibility to ciprofloxacin have been confirmed previously (Fendukly et al., 2003; Heisig, 1996; Trees et al., 1998). In addition, the isolate was roughly tested for CCCP-sensitive efflux to exclude the possible role of an efflux pump in reducing the susceptibility of EIEC O164 strain RIMD05091045 to ciprofloxacin. The efflux experiments showed no change in the MIC of ciprofloxacin $\left(2 \mu \mathrm{g} \mathrm{ml}^{-1}\right)$ in the presence of CCCP. Hence, an efflux pump may have no role in the reduced susceptibility to ciprofloxacin in this strain. 


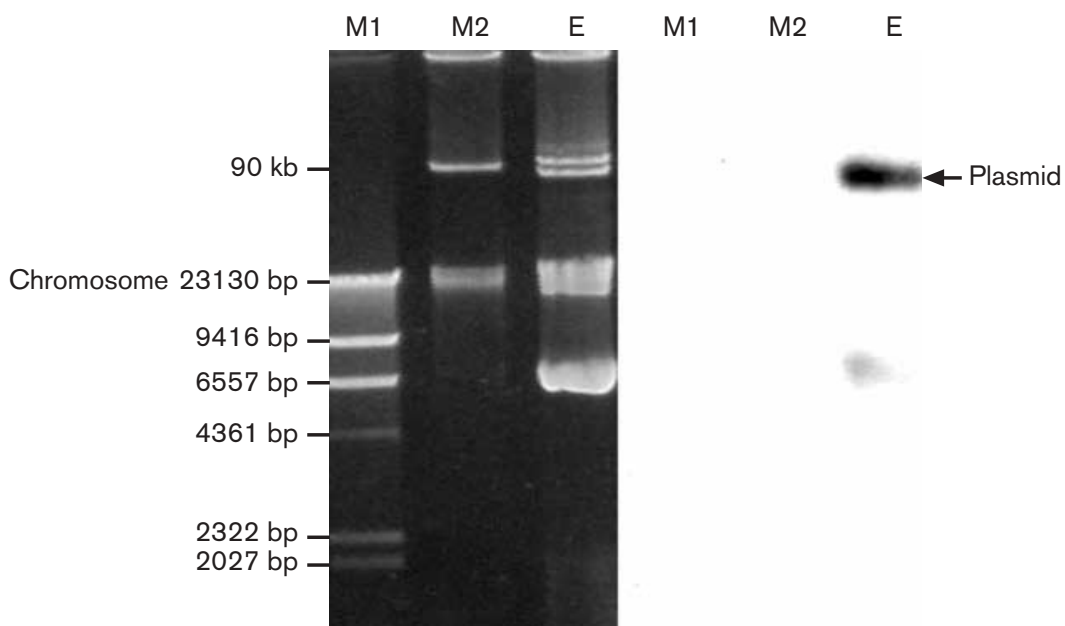

\begin{abstract}
Fig. 3. Agarose gel electrophoresis (left panel) and Southern blot hybridization (right panel) of the plasmid-encoded class 1 integron of EIEC O164 strain RIMD05091045. Lanes M1 and M2 contain $\lambda$ DNA digested with Hindlll and Salmonella typhimurium LT2 plasmid (90 kb), respectively, as size markers. Lane $\mathrm{E}$ contains the plasmids of EIEC 0164 strain RIMD05091045, including part of its chromosome. The hybridization signal is in a plasmid of less than $90 \mathrm{~kb}$.
\end{abstract}

Although complementation and transformation studies need to be performed to confirm the role of this novel mutation, our results suggest that it may be responsible for the reduced susceptibility to ciprofloxacin in EIEC O164 strain RIMD05091045. Furthermore, since several reports have described resistance mutations outside the QRDRs of gyrA (Chaudhry et al., 2002; Fournier \& Hooper, 1998; Ince \& Hooper, 2000) and parC (Bebear et al., 2003; Chaudhry et al., 2002; Fendukly et al., 2003; Trees et al., 1998), our finding supports the concept that the size of the QRDRs may require revision and should be expanded.

\section{Conclusion}

In this study, we characterized the genetic bases of the MDR phenotype in EIEC O164 strain RIMD05091045 in relation to its class 1 integron, $\beta$-lactamase-encoding genes and mutations in the QRDRs of DNA gyrase and topoisomerase IV. The class 1 integron of EIEC O164 strain RIMD05091045 is located on a transferable plasmid and the resistance phenotype was transferred to a laboratory strain of $E$. coli HB101. A TEM-1 $\beta$-lactamase gene was found to be responsible for ampicillin resistance in this strain. In addition, we detected a new point mutation outside the QRDR of $\operatorname{parC}$ of topoisomerase IV. Therefore, we agree with other researchers who have suggested that the range of the QRDRs should be expanded.

\section{ACKNOWLEDGEMENTS}

This work was supported by a grant-in-aid for scientific research to T.S. from the Ministry of Education, Culture, Sports, Science and Technology of Japan.

\section{REFERENCES}

Ahmed, A. M. \& Shimamoto, T. (2004). A plasmid-encoded class 1 integron carrying sat, a putative phosphoserine phosphatase gene and aadA2 from enterotoxigenic Escherichia coli O159 isolated in Japan. FEMS Microbiol Lett 235, 243-248.

Bebear, C. M., Renaudin, H., Charron, A., Clerc, M., Pereyre, S. \&
Bebear, C. (2003). DNA gyrase and topoisomerase IV mutations in clinical isolates of Ureaplasma spp. and Mycoplasma hominis resistant to fluoroquinolones. Antimicrob Agents Chemother 47, 3323-3325.

Bopp, C. A., Brenner, F. W., Wells, J. G. \& Stockbine, N. A. (1999). Escherichia, Shigella, and Salmonella. In Manual of Clinical Microbiology, 7th edn, pp. 459-474. Edited by P. R. Murray and others. Washington, DC: American Society for Microbiology.

Bradford, P. A. (2001). Extended-spectrum $\beta$-lactamases in the 21 st century: characterization, epidemiology, and detection of this important resistance threat. Clin Microbiol Rev 14, 933-951.

Chaudhry, U., Ray, K., Bala, M. \& Saluja, D. (2002). Mutation patterns in gyrA and parC genes of ciprofloxacin resistant isolates of Neisseria gonorrhoeae from India. Sex Transm Infect 78, 440-444.

Fendukly, F., Karlsson, I., Hanson, H. S., Kronvall, G. \& Dornbusch, K. (2003). Patterns of mutations in target genes in septicemia isolates of Escherichia coli and Klebsiella pneumoniae with resistance or reduced susceptibility to ciprofloxacin. APMIS 111, 857-866.

Fournier, B. \& Hooper, D. C. (1998). Mutations in topoisomerase IV and DNA gyrase of Staphylococcus aureus: novel pleiotropic effects on quinolone and coumarin activity. Antimicrob Agents Chemother 42, $121-128$.

Gassama, A., Aidara-Kane, A., Chainier, D., Denis, F. \& Ploy, M. C. (2004). Integron-associated antibiotic resistance in enteroaggregative and enteroinvasive Escherichia coli. Microb Drug Resist 10, 27-30.

Harris, J. R., Mariano, J., Wells, J. G., Payne, B. J., Donnell, H. D. \& Cohen, M. L. (1985). Person-to-person transmission in an outbreak of enteroinvasive Escherichia coli. Am J Epidemiol 122, 245-252.

Heisig, P. (1996). Genetic evidence for a role of parC mutations in development of high-level fluoroquinolone resistance in Escherichia coli. Antimicrob Agents Chemother 40, 879-885.

Ince, D. \& Hooper, D. C. (2000). Mechanisms and frequency of resistance to premafloxacin in Staphylococcus aureus: novel mutations suggest novel drug-target interactions. Antimicrob Agents Chemother 44, 3344-3350.

Jones, M. E., Peters, E., Weersink, A. M., Fluit, A. \& Verhoef, J. (1997). Widespread occurrence of integrons causing multiple antibiotic resistances in bacteria. Lancet 349, 1742-1743.

Lévesque, C., Piché, L., Larose, C. \& Roy, P. H. (1995). PCR mapping of integrons reveals several novel combinations of resistance genes. Antimicrob Agents Chemother 39, 185-191.

Livermore, D. M. (1995). $\beta$-Lactamases in laboratory and clinical resistance. Clin Microbiol Rev 8, 557-584. 
Marier, R., Wells, J. G., Swanson, R. C., Callahan, W. \& Mehlman, I. J. (1973). An outbreak of enteropathogenic Escherichia coli foodborne disease traced to imported French cheese. Lancet 2, 1376-1378.

Maynard, C., Fairbrother, J. M., Bekal, S., Sanschagrin, F., Levesque, R. C., Brousseau, R., Masson, L., Larivière, S. \& Harel, J. (2003). Antimicrobial resistance genes in enterotoxigenic Escherichia coli O149:K91 isolates obtained over a 23-year period from pigs. Antimicrob Agents Chemother 47, 3214-3221.

Nataro, J. P. \& Kaper, J. B. (1998). Diarrheagenic Escherichia coli. Clin Microbiol Rev 11, 142-201.

NCCLS (2002). Performance Standards for Antimicrobial Susceptibility Testing, 12th Informational Supplement, Approved Standard M100S12, NCCLS. Wayne, PA: National Committee for Clinical Laboratory Standards.

Orskov, F. \& Orskov, I. (1984). Serotyping of Escherichia coli. Methods Microbiol 14, 43-112.

Piddock, L. J. V. (1999). Mechanisms of fluoroquinolone resistance: an update 1994-1998. Drugs 58, 11-18.

Rowe-Magnus, D. A. \& Mazel, D. (2001). Integrons: natural tools for bacterial genome evolution. Curr Opin Microbiol 4, 565-569.

Rowe-Magnus, D. A., Guerout, A. M. \& Mazel, D. (2002). Bacterial resistance evolution by recruitment of super-integron gene cassettes. Mol Microbiol 43, 1657-1669.

Sambrook, J. \& Russell, D. (2001). Molecular Cloning: a Laboratory Manual, 3rd edn. Cold Spring Harbor, NY: Cold Spring Harbor Laboratory.

Snyder, J. D., Wells, J. G., Yashuk, J., Puhr, N. \& Blake, P. A. (1984). Outbreak of invasive Escherichia coli gastroenteritis on a cruise ship. Am J Trop Med Hyg 33, 281-284.

Taylor, D. N., Echeverria, P., Pal, T., Sethabutr, O., Sailborisuth, S. Sricharmom, S., Rowe, B. \& Cross, J. (1986). The role of Shigella ssp. enteroinvasive Escherichia coli, and other enteropathogens as causes of childhood dysentery in Thailand. J Infect Dis 153, 1131-1138.
Todorova, K., Bratoeva, M. \& Daneva, M. (1990). Characterization of enteroinvasive Escherichia coli serotype $\mathrm{O} 164$ by means of plasmid analysis and virulence assay. J Basic Microbiol 30, 451-454.

Toma, C., Lu, Y., Higa, N., Nakasone, N., Chinen, I., Baschkier, A., Rivas, M. \& Iwanaga, M. (2003). Multiplex PCR assay for identification of human diarrheagenic Escherichia coli. J Clin Microbiol 41, 2669-2671.

Trees, D. L., Sandul, A. L., Whittington, W. L. \& Knapp, J. S. (1998). Identification of novel mutation patterns in the parC gene of ciprofloxacin-resistant isolates of Neisseria gonorrhoeae. Antimicrob Agents Chemother 42, 2103-2105.

Webber, M. A. \& Piddock, L. J. V. (2001). Absence of mutations in $\operatorname{marR} A B$ or soxRS in acrB-overexpressing fluoroquinolone-resistant clinical and veterinary isolates of Escherichia coli. Antimicrob Agents Chemother 45, 1550-1552.

Webber, M. A. \& Piddock, L. J. V. (2003). The importance of efflux pumps in bacterial antibiotic resistance. J Antimicrob Chemother 51, 9-11.

Weill, F.-X., Demartin, M., Tande, D., Espie, E., Rakotoarivony, I. \& Grimont, P. A. D. (2004). SHV-12-like extended-spectrum- $\beta$-lactamase-producing strains of Salmonella enterica serotypes Babelsberg and Enteritidis isolated in France among infants adopted from Mali. J Clin Microbiol 42, 2432-2437.

WHO (1995). Guidelines for the Control of Epidemics due to Shigella dysenteriae 1. Geneva: World Health Organization, Publication no. $\mathrm{WHO} / \mathrm{CDR} / 95.4$

Yamamura, K., Sumi, N., Egashira, Y., Fukuoka, I., Motomura, S. \& Tsuchida, R. (1992). Food poisoning caused by enteroinvasive Escherichia coli (O164:H-) - a case in which the causative agent was identified (Japanese). J Jpn Assoc Infect Dis 66, 761-768.

Zhao, S., White, D. G., Ge, B., Ayers, S., Friedman, S., English, L., Wagner, D., Gaines, S. \& Meng, J. (2001). Identification and characterization of integron-mediated antibiotic resistance among shiga toxinproducing Escherichia coli isolates. Appl Environ Microbiol 67, 15581564. 